\title{
Causes of childhood deafness in Pukhtoonkhwa Province of Pakistan and the role of consanguinity
}

\author{
M SAJJAD*, A A KhatTAK†, J E G Bunn
}

\begin{abstract}
Background: Deafness is the hidden disability of childhood, and leads to poor educational and employment prospects. There is little published information on deafness in Pakistan. Profound hearing impairment is more prevalent in countries where consanguineous marriages are common, such as Pakistan. This study aimed to assess causes of childhood deafness and association with parental consanguinity, within deaf and hearing children in the Peshawar district of Pukhtoonkhwa Province, Pakistan.

Methods: One hundred and forty deaf children were identified from two schools for deaf children within the Peshawar district. These children were assessed via audiology, otoscopic examination, case note review and parental history, in order to attempt to ascertain the cause of their deafness. Two hundred and twenty-one attendees at a local immunisation clinic (taken as representative of the local childhood population) were also screened for hearing impairment. Parents of both groups of children were assessed by interview and questionnaire in order to ascertain the mother and father's family relationship (i.e. whether cousins or unrelated).

Results: Of the 140 deaf school pupils, 92.1 per cent were profoundly hearing impaired and 7.9 per cent were severely hearing impaired. All these children had bilateral sensorineural hearing loss. A possible cause of deafness was identified in only six of these children. Parental consanguinity (i.e. first or second cousins) was established for 86.4 per cent of deaf school pupils and 59.7 per cent of immunisation clinic attendees. None of the control children were identified as having a hearing problem.

Conclusion: The prevalence of parental consanguinity was significantly higher in deaf children compared with non-hearing impaired children. However, the study also confirmed a high rate of consanguinity within the general Peshawar community. In this setting, prevention of consanguineous unions is the only means of reducing levels of congenital hearing impairment. The current levels of hearing disability represent both a prominent public health problem and an important, potentially preventable childhood disability.
\end{abstract}

Key words: Sensorineural Deafness; Pakistan; Consanguinity

\section{Introduction}

Deafness is the hidden disability of childhood, and leads to poor educational and employment prospects. Deafness is classified as hearing loss over the speech frequencies $500 \mathrm{~Hz}, 1 \mathrm{KHz}$ and $4 \mathrm{KHz}$; an average hearing loss greater than $41 \mathrm{~dB}$ is regarded as disabling. Hearing loss is classified as follows: mild, 26-40 dB; moderate, 41-60 dB; severe, 61-80 dB; and profound $>80 \mathrm{~dB}$.

There is little published information on the causes of deafness in Pakistan, especially in the Pukhtoonkhwa Province, which includes the city of Peshawar.

Review of the literature revealed few published papers on deafness in Pakistan. It is known that one in 1000 children worldwide are born profoundly deaf. ${ }^{1}$ In Pukhtoonkhwa Province, there are several schools for deaf children, funded by voluntary organisations and the government. If these children's causes of deafness could be identified, this would indicate the most likely causes of deafness in the community. It is already established that 50 per cent of the causes of deafness are preventable. $^{2}$

There is evidence that profound hearing impairment is more prevalent in countries where consanguineous marriages are common. ${ }^{3}$ In this region of Pakistan, the prevalence of consanguinity is reported to be 60 per cent. ${ }^{4}$ The aim of this study was to

From the *Child and Reproductive Health Group, Liverpool School of Tropical Medicine, UK, the †Department of Paediatrics, Lady Reading Hospital, Peshawar, Pakistan, the $\ddagger$ Department of Paediatrics, College of Medicine, Blantyre, Malawi, and the $\S$ Audiology Department, Oldham Primary Care Trust, UK.

Accepted for publication: 29 August 2007. First published online 21 April 2008. 
establish the common causes of deafness within the studied region, and to determine any relationship with parental consanguinity.

Consanguineous marriages are traditional in many Asian, African and South American communities. These communities have practised this tradition both when living in their native country and when settled in other countries. The degree of consanguinity varies from first cousins to more distant relatives, and its prevalence varies from culture to culture. Consanguineous marriages are most prevalent in Arab countries, followed by India, Japan, Brazil and Israel. Consanguineous unions are most common in lower educational and socioeconomic groups, amongst the traditionally religious, and in those marrying young.

In Europe and North America, marriage between close relatives is looked upon unfavourably. However, in other parts of the world, particularly in the Muslim community, 20-50 per cent of all marriages are consanguineous. In one Indian study, ${ }^{5}$ consanguinity was described as 'marriages between second cousins or more closely related individuals'. This definition complies with that used by the National Society of Genetic Counselors. ${ }^{6}$

Consanguinity generally increases prereproductive mortality in offspring; crude mortality increases in proportion to the degree of inbreeding. Many studies have demonstrated that consanguinity is associated with a significant increase in disease rates, particularly in the Muslim community. ${ }^{7}$ Epidemiological studies usually measure inbreeding effects in terms of genetic load, which is not readily translatable into morbidity and mortality figures. Confounding is the most difficult problem in these studies, because of the difficulty in selecting nonrelated controls. For this reason, consanguinity studies should be subject to both genetic and epidemiological analysis, in order to illuminate the role of genetic factors and the relevance of consanguinity to disease and public health. ${ }^{8}$

Many studies have shown that the offspring of consanguineous marriages have a greater incidence of autosomal recessive diseases, including hearing impairment. ${ }^{9}$ In addition, the great majority of hereditary deafness is caused by single gene, autosomal recessive inheritance. ${ }^{10}$

A Saudi Arabian study involving 9500 subjects reported the frequency of consanguineous marriage, together with the prevalence of hearing impairment amongst the siblings of consanguineous and nonrelated pairings. This study demonstrated a definite association between parental consanguinity and childhood hearing impairment prevalence. ${ }^{3}$

In 2005, Mytton and Mackenzie ${ }^{11}$ published data from Oldham in Greater Manchester indicating a very high rate of profound deafness in the local Pakistani community.

\section{Genetic basis of deafness}

Congenital deafness is a complex disease involving both genetic and environmental factors. Studies have demonstrated the involvement of a genetic component in the development of deafness in approximately 60 per cent of cases. Non-syndromic hearing impairment accounts for 80 per cent of cases of hereditary deafness. Hearing impairment exhibits all forms of inheritance, including autosomal dominant, autosomal recessive, $\mathrm{X}$-linked recessive and mitochondrial. Moreover, mutations in the same single gene have been shown to be responsible for both autosomal dominant and autosomal recessive forms of hearing impairment (e.g. connexion 26). ${ }^{12}$ Many genes are involved in both syndromic and nonsyndromic hearing loss. ${ }^{13}$

In the past few years, there has been rapid progress in the identification of genes involved in hearing impairment, improving our understanding of the mechanisms of hearing. A single locus, DFNB1, has been found to contribute to the majority of cases of autosomal recessive, non-syndromic hearing impairment. ${ }^{14,15}$

In 1997, Gasparini et al. ${ }^{16}$ undertook a genetic linkage study using four microsatellite markers linked to the DFNB1 locus, in a total of 48 independent Mediterranean families. These authors demonstrated that the DFNB1 locus played a role in the majority of these families with non-syndromic, neurosensory, autosomal recessive deafness.

In 2004, Nance and Kearsey ${ }^{17}$ demonstrated by computer simulation that assortative mating can accelerate dramatically the genetic response and aid understanding of the effect of consanguinity.

\section{Prevalence of consanguinity in Pakistan}

It is known that consanguineous marriages are strongly preferred in many parts of western and southern Asia and north Africa. ${ }^{5}$ Unfortunately, limited information on the topic is available in the mainstream demographic literature. However, such information is available from demographic and health surveys in some countries, including India, Pakistan and Bangladesh. ${ }^{18}$

The Pakistan Demographic and Health Survey 1990-1991 showed that more than 50 per cent of married women aged 15-49 years were married to their first cousins. The inclusion of second cousins raised the percentage of consanguineous marriages from 50.3 to 61.2 per cent. Another 1.3 per cent of the women surveyed were married to other relations; the remainder (over 37 per cent) were married to non-relatives. Differences became apparent between rural and urban areas within different provinces in Pakistan. In urban areas, 51.3-53.1 per cent of marriages were consanguineous; in rural areas, this figure rose to 65.6-66.9 per cent. The percentage of marriages involving first cousins was higher in the provinces of Baluchistan (53 per cent) and Punjab (54.4 per cent). In addition, the Pakistan Demographic and Health Survey found that child mortality and morbidity rates were significantly higher in the families of consanguineous parents. ${ }^{19}$

In one study from Pakistan, ${ }^{20}$ the prevalence and sociodemographic correlates of consanguineous marriages in Karachi city were compared with data from the national 1990-1991 Pakistan Demographic 
and Health Survey. The authors found that approximately 60 per cent of marriages were consanguineous, over 80 per cent of which were between first cousins. Using the coefficient of inbreeding $(\mathrm{F})$, representing the probability that a person with two identical genes received both genes from one common ancestor, the authors calculated values for this coefficient of 0.0316 and 0.0331 (in the present generation) for the Karachi and Pakistan Demographic and Health Survey data sets, respectively. In addition, the prevalence of consanguineous unions remained unchanged, comparing the preceding three to four decades and different populations. The prevalence of consanguineous marriages was higher where women were illiterate or had only primary level education, or were first or second generation migrants from rural areas of Pakistan. This finding suggested that consanguinity was an issue of social concern, regarding the well-being both of daughters and of their families.

\section{Hearing impairment in children}

Children with hearing impairment may be seriously disabled. Deaf children are often poorly educated and lack normal speech development which limits their ability to socialise, resulting in social and occupational problems in later life. There are a number of risk factors likely to make congenital deafness more common in developing countries, in addition to consanguinity. Poor socioeconomic conditions, a high incidence of infectious diseases, poor vaccination coverage and deficient obstetric services all contribute to an increased risk of brain damage at birth. In addition, there is a lack of reliable, standardised epidemiological data on the prevalence and causes of hearing impairment in developing countries.

Studies classifying congenital deafness by cause have used a variety of classification systems, which makes comparison across studies difficult. Hearing loss severity has usually been classified as severe (profound), moderate or mild, but the thresholds for each category have varied considerably.

Hearing loss is a significant sensory disability, and includes impairment due to environmental factors (e.g. acoustic trauma, ototoxicity, and viral and bacterial infections). Hearing impairment can be classified as prelingual versus postlingual, conductive versus sensorineural, syndromic versus nonsyndromic and genetic versus acquired. ${ }^{12}$

In developing countries, the World Health Organization recommends that children's hearing should be screened at least at school entry, using a simple audiometer, and that the external ear be inspected for the presence of discharge. ${ }^{21}$ In an ideal world, neonatal hearing screening should be encouraged.

\section{Methods}

The present study was conducted within schools for deaf children in the Peshawar district of Pukhtoonkhwa Province, Pakistan. In order to obtain a good cross-section of the local community as a control, we also approached parents of children attending for measles-mumps-rubella vaccination at the immunisation clinic of the Lady Reading Hospital, Peshawar. The Lady Reading Hospital postgraduate medical institute is a tertiary level hospital and one of the biggest hospitals in the province. The hospital runs an open access immunisation clinic from Monday to Saturday each week. Children from the city of Peshawar attend this clinic. We assumed that these children's parents constituted a representative sample of married adults within the community. Thus, assessing these parents was a logistically feasible way of estimating consanguinity rates within the local community.

There are two schools for deaf children within the main city of Peshawar. These schools have soundproof rooms for audiometry, and a qualified audiologist is employed for hearing assessment. The equipment is old but reliable.

Ethical approval for the study was obtained from the Liverpool School of Tropical Medicine and later from the Lady Reading Hospital.

\section{Study design}

We undertook a cross-sectional survey of 140 deaf children attending deaf school, in order to identify the cause of their deafness and also parental consanguinity. We also assessed hearing and parental consanguinity amongst 220 control children randomly selected from the Lady Reading Hospital open access immunisation clinic. We then conducted a case-control analysis to investigate the association between profound childhood deafness and parental consanguinity.

\section{Sample size}

We assumed a prevalence of consanguinity of up to 60 per cent in the normal community and of approximately 76 per cent in parents of deaf school pupils. Therefore, we calculated that we required 140 cases (i.e. deaf school pupils) and 220 controls (i.e. children identified from the immunisation clinic, with no history of deafness) in order to generate a study power of 80 per cent and to detect a difference at 95 per cent confidence intervals.

\section{Procedures}

Study procedures comprised: pure tone audiometry and otoscopy of children; modified behavioural testing of babies; and a parental questionnaire and an interview of both parents in the majority of cases. Audiometric results were recorded in the deaf children's school clinic records.

\section{Data collection and analysis}

Causes of deafness were determined by the main author (MS) and double-entered into the Epi Info ${ }^{\mathrm{TM}}$ database. Data on consanguinity were analysed using two by two tables and chi-square testing. The prevalence of consanguinity was reported with 95 per cent confidence intervals.

Cases were identified from two schools for deaf children in Peshawar city, and controls from the 
Lady Reading Hospital immunisation clinic, Peshawar.

Urban deaf schools were chosen so that parents or guardians would be easily available to advise their child's cause of deafness. Interviews were conducted with the help of a questionnaire (translated into Urdu by the main author (MS), with responses translated back into English by another author (IM)).

Permission was obtained to access the local hospital's medical notes, in order to obtain any relevant information (e.g. birth records for cases of drug ototoxicity).

Children from five to 14 years cooperated well with pure tone audiometry. Hearing thresholds were established by the main author (MS) at the speech frequencies, using techniques described by the British Society of Audiology. Audiometry training was given prior to the study by another author (IM). Ear examinations were performed otoscopically and the findings entered into a questionnaire.

In order to establish whether consanguinity was associated with deafness, the prevalence of parental consanguinity amongst deaf children was compared with that amongst children attending the immunisation clinic. Children's medical histories (for both cases and controls) were obtained by interviewing their parents. Data requested included the parent's place of residence, community and whether the marriage was consanguineous. When the latter answer was in the affirmative, the exact family relationship was established (e.g. first or second cousins). Parents were asked about the health of any siblings, and whether any other family members were deaf.

Control children were screened for hearing impairment within the immunisation clinic. This involved presentation of loud sounds and observation of the child's response and their aural-palpebral reflex. None of the control children were suspected of having severe hearing loss, based on parental report or prior screening.

Consent was obtained in writing, or by thumbprint, using consent forms translated into the local language.

\section{Results}

A total of 361 children were studied: 140 cases from schools for deaf children and 221 controls from the immunisation clinic. The case group comprised 70 boys and 70 girls, with mean ages of 11.0 and 10.2 years, respectively. The control group comprised 49.3 per cent boys and 50.7 per cent girls, with a mean group age of 0.8 years.

\section{Consanguinity}

The family relationships of parents of case and control group children were as follows (Tables I and II).

Case group. Of these 140 children, 103 (73.6 per cent) had parents who were first cousins, 18 (12.9 per cent)
TABLE I

PARENTAL CONSANGUINITY AMONGST CASE AND CONTROL GROUP CHILDREN: FIRST COUSINS

\begin{tabular}{lcr}
\hline Sample group & \multicolumn{2}{c}{ Consanguinity degree $(n(\%))$} \\
\cline { 2 - 3 } & 1st cousins & Unrelated \\
\hline Case & $103(73.6)$ & $19(13.6)$ \\
Control & $90(40.7)$ & $89(40.3)$ \\
\hline
\end{tabular}

Analysis of difference between case and control group: chi-square $=35.3 ; \quad p=0.0000 ; 95 \%$ confidence intervals $1.42-1.98$

second cousins and 19 (13.6 per cent) unrelated. A parental family relationship more distant than second cousins was defined as unrelated. Thus, parental consanguinity (i.e. first or second cousins) amongst deaf children was 86.4 per cent.

Control group. Of the children in this group, 59.7 per cent had consanguineous parents.

The difference in the proportion of consanguineous parents, comparing case and control group children, was significant (chi-square, $p=0.0001$ ).

\section{Hearing impairment}

In the 220 case group children, pure tone audiometry (binaural average hearing threshold) revealed that 129 (92.1 per cent, 95 per cent confidence intervals (CIs) 86.4-96.0) were profoundly deaf (i.e. $>80 \mathrm{~dB}$ hearing loss) and 11 (7.9 per cent, 95 per cent CIs 4.0-13.6) were severely deaf (i.e. 61$80 \mathrm{~dB}$ hearing loss). All case group children had bilateral sensorineural hearing loss. None of these children were found to have mild or moderate hearing loss.

Possible causes of deafness were identified in six case group children, and these are summarised in Table III. Two children had chronic suppurative otitis media (CSOM). One case of Wardenberg's syndrome was identified. Common infections such as mumps, measles and meningitis were all identified as potential causes of deafness. In the remaining 134 (96 per cent) children, the cause of deafness was not identified on history or examination. None of the control children were identified as having a hearing problem.

There was no significant difference in parents' financial status, socioeconomic status or educational attainment, comparing the case and control groups.

TABLE II

PARENTAL CONSANGUINITY AMONGST CASE AND CONTROL GROUP CHILDREN: FIRST AND SECOND COUSINS

\begin{tabular}{lcc}
\hline Sample group & \multicolumn{2}{c}{ Consanguinity degree $(n(\%))$} \\
\cline { 2 - 3 } & 1st or 2nd cousins & Unrelated \\
\hline Case & $121(86.4)$ & $19(13.6)$ \\
Control & $132(59.7)$ & $89(40.3)$ \\
\hline
\end{tabular}

Analysis of difference: chi-square $=27.88 ; p=0.00001 ; 95 \%$ confidence intervals 1.27-1.64. 
TABLE III

CASE GROUP CHILDREN: IDENTIFIED CAUSES OF HEARING IMPAIRMENT

\begin{tabular}{lrc}
\hline Cause & \multicolumn{2}{c}{ Cases } \\
\cline { 2 - 3 } & $n$ & $\%$ \\
\hline Measles & 2 & 1.4 \\
Meningitis & 3 & 2.1 \\
Mumps & 1 & 1 \\
Wardenburg's syndrome & 3 & 2.1 \\
Unknown & 134 & 95.7 \\
Total & 143 & 100 \\
\hline
\end{tabular}

\section{Discussion}

\section{Consanguinity and hearing impairment in Pakistan}

Consanguineous marriages are preferred in many Asian countries. This study assessed the causes of childhood deafness and any possible association of consanguinity as a risk factor. Data on the prevalence of parental consanguinity were obtained from pupils of two schools for deaf children and from immunisation clinic attendees, within Peshawar district, Pukhtoonkhwa Province, Pakistan.

Only two deaf children were found to have CSOM; this is to be expected where children are well fed and cared for.

Ideally, we would have matched the case and control groups in terms of sex and age. However, this was logistically difficult because it was not possible to obtain permission to conduct the study within a normal school. Even if this had been possible, not all children in this community attended school, so such a sample would have been suboptimal. Therefore, it was decided to assess attendees at an immunisation clinic, as this context enabled hearing testing of children and easy access to parents for interviews.

Children's parents were questioned in order to establish the mother and father's family relationship (i.e. first cousins, second cousins or unrelated). In the control group, the reported rate of parental consanguinity was assumed to reflect that within the local community. In the control group, parental consanguinity was reported for 59.7 per cent of children. This is similar to the 62.7 per cent reported in the Pakistan Demographic and Health Survey 1990$1991,{ }^{18}$ although higher than the 38.8 per cent reported in 1994 by Afzal et al. ${ }^{19}$ for Pukhtoonkhwa Province. This may suggest that rates of consanguinity are rising, or that changes in urban-rural differences may be important ${ }^{20}$ indeed, Afzal et al. reported a higher prevalence of consanguinity in rural areas.

\section{Effect of socioeconomic and educational status on hearing impairment}

In 2002, Rao et al. $^{21}$ reported that the prevalence of hearing impairment in India was significantly lower among children of high socioeconomic status $(p=0.0036)$. We found that the socioeconomic and educational status of children's parents did not differ significantly, comparing the case and control groups. However, it is not possible to ignore the importance of parental socioeconomic and educational status, regarding childhood hearing impairment. It has been suggested that the children of low socioeconomic status families will suffer frequent ear infections and have poorer access to health facilities, and will thus be at greater risk of hearing impairment, especially in resource-poor countries. ${ }^{22}$ However, such children are less likely to attend a school for the profoundly deaf. In addition, we found that all case group children had sensorineural rather than conductive deafness.

\section{Causes of hearing impairment, and consanguinity as a possible risk factor}

In a study conducted by Elahi et al. ${ }^{23}$ in Pakistan, a total of 607 children of school age were screened and examined for hearing impairment. These authors reported that 50 per cent of all hearing loss detected was conductive in nature. The risk factors most associated with conductive hearing loss were otorrhoea and multiple (i.e. more than five) ear infections. In cases of severe sensorineural hearing loss, 70 per cent were associated with consanguineous marriages. Almost no cases of hearing loss were attributed to measles, mumps, rubella and with TORCH [toxoplasmosis, rubella cytomegalovirus and herpes] infections. In our study, only a small number of deaf children reported a past history of measles (1.4 per cent), mumps (0.7 per cent) or meningitis (2.1 per cent). However, in most of these children no obvious cause of severe hearing loss could be established (Table III). In our study, it would appear that 93.7 per cent of cases of severe hearing loss were due to autosomal recessive gene inheritance and 2 per cent to autosomal dominant gene inheritance. In the future, it is expected that deafness will be less frequently attributed to unknown causes, due to the availability of molecular genetic testing. 10

- In the developing world, deafness is the hidden disability of childhood and leads to poor educational and employment prospects

- There is evidence that profound hearing impairment is more prevalent in countries where consanguineous marriages are common

- This study investigated the aetiology of deafness amongst 140 children identified from two schools for deaf children in the Peshawar district of Pukhtoonkhwa Province, Pakistan

- Consanguinity appears to be the dominant cause of profound sensorineural deafness in Pakistan - an important, potentially preventable childhood disability

In a study of Saudi Arabian children in Riyadh by Zakzouk, hearing impairment was significantly 
more prevalent in children whose parents were either first cousins (16.14 per cent, $p<0.001)$ or otherwise related (12.42 per cent, $p<0.01)$, compared with children whose parents were unrelated. ${ }^{3}$ These results support our own findings of a possible association between consanguineous marriages and childhood hearing loss. In addition, it is customary to exclude marriages between individuals less closely related than second cousins, when reporting the prevalence of consanguineous marriages. Second cousins inherit 1/32 of their genes from a common ancestor. This means that offspring inherit identical genes at 1/64 (1.56 per cent) of all loci. This variable is expressed as the coefficient of inbreeding $(\mathrm{F})$. For the progeny of second cousins, $F=0.0156$; for the progeny of first cousins, $\mathrm{F}=0.0625$. In the latter situation, offspring inherit identical genes at 6.25 per cent of all loci. For the progeny of an uncle and a niece, or of double first cousins, $\mathrm{F}=0.125$. $^{4}$

The Saudi Arabian study ${ }^{3}$ recorded a slightly higher number of sensorineural hearing loss cases due to possible environmental causes (e.g. meningitis or mumps). In our study, hereditary and unknown causes appeared to be the predominant aetiological factors; hearing loss was generally of unknown aetiology and had been present from birth, and no exogenous causes could be established. These findings suggest that such cases could be classified as congenital deafness. According to Fortnum and Davis's definition, hearing impairment should be considered congenital unless there is evidence of progressive, late onset or exogenous causes. $^{24}$

It has been shown that there is a significant deaf population within the Pakistani community in Oldham, near Manchester, UK. ${ }^{11}$ This community originated from the north-west of Pakistan, in the region of the border with Kashmir, where consanguinity is common.

\section{Conclusions}

This study found a significant difference in the prevalence of parental consanguinity in children attending deaf schools, compared with the normal population. It clearly demonstrated an association between parental consanguinity and severe to profound childhood hearing loss. The study confirmed a high rate of consanguinity within the Peshawar community; the resulting levels of hearing disability represent a prominent public health problem in this community. The study also described the role of environmental causes in hearing impairment.

Prevention is the only means to reduce the prevalence of congenital hearing impairment. This can be achieved by genetic counselling of individuals and families at high risk. A well organised counselling programme to create awareness of the adverse effects of consanguineous marriages could be helpful in reducing the prevalence of deafness within this community. The Saudi Arabian government, aware of Zakzouk's findings, have not been encouraging first cousin marriages for the last 10 years, and already there has been a reduction in consanguineous marriages and children born with hearing disability.

Consanguinity appears to be the dominant cause of profound sensorineural deafness in Pakistan an important, potentially preventable childhood disability.

\section{References}

1 Newton V. Aetiology of bilateral sensori neural hearing loss in young children. J Laryngol Otol 1985;10: $1-57$

2 World Health Organization 1996. Prevention of Deafness Survey Protocol. Geneva: World Health Organization, 1992

3 Zakzouk S. Consanguinity and hearing impairment in developing countries: a custom to be discouraged. J Laryngol Otol 2002;116:811-16

4 Hussain R, Bittles AH. The prevalence and demographic characteristics of consanguineous marriages in Pakistan. J Biosoc Sci 1998;30:261-75

5 Bittles AH. The role and significance of consanguinity as demographic variables. Dev Rev 1994;20:561-84

6 Bennett RL. Leading voices and the power of one. J Genetic Counselling 2003;12:97-107

7 Shanky S, Albalkhail B, Soliman N. An epidemiological study of childhood disability in Jeddah, Saudi Arabia. Pediatric and Perinatal Epidemiology 2002;16:61-6

8 Khlat M, Khoury M. Inbreeding and diseases: demographic, genetic, and epidemiologic perspectives. Epidemiol Rev 1991;13:28-41

9 Stevenson AC, Cheeseman EA. Hereditary deaf mutism with particular reference to Northern Ireland. Ann Hum Genet 1956;20:177-231

10 Smith RJH. Medical diagnosis and treatment of hearing loss in children. In: Otolaryngology - Head and Neck Surgery. Cummings CW, ed. St Louis: CV Mosby, 1986;4: 3225-46

11 Mytton J, Mackenzie I. Permanent childhood deafness in Oldham. J Public Health 2005;27:298-302

12 Morton NE. Genetic epidemiology of hearing impairment. Ann N Y Acad Sci 1991;630:16-31

13 Cohen MM, Gorlin RJ. Epidemiology, etiology, and genetic patterns. In: Hereditary Hearing Loss and Its Syndromes. Gorlin RJ, Toriello HV, Cohen MM Jr, eds. Oxford: Oxford University Press, 1995;9-21

14 Guilford P, Ben Arab S, Blanchard S, Levilliers J, Weissenbach J, Belkahia A et al. A non syndromic form of neurosensory, recessive deafness maps to the pericentromeric region of chromosome 13q. Nature Genet 1994;4: $24-8$

15 Brown KA, Janjua AH, Karbani G, Parry G, Noble A, Crockford G et al. Linkage studies of non-syndromic recessive deafness (NSRD) in a family originating from the Mirpur region of Pakistan maps DFNB1 centromeric to D13S175. Hum Mol Genet 1996;5:169-73

16 Gasparini P, Estivill X, Volpini V, Totaro A, CastellviBel S, Govea N, et al. Linkage of DFNB1 to nonsyndromic neuro-sensory autosomal-recessive deafness in Mediterranean families. Eur J Hum Mol Genet 1997; 5:83-8

17 Nance WE, Kearsey MJ. Relevance of connexin deafness (DFNB1) to human evolution. Am J Hum Genet 2004;74: 1081-7

18 Ahmed T, Ali SM, Aliaga A, Arnold F, Ayub M, Bhatti MH. Pakistan demographic and health survey 1990-91. Islamabad: National Institute of Population Studies, 1992;9-13

19 Afzal M. Consequences of consanguinity in cognitive behaviour. Behaviour Genetics 1988;18:593-594

20 Hussain R, Bittles AH. Assessment of association between consanguinity and fertility in Asian population. $J$ Health Popul Nutr 2004;22:1-12 
21 Rao RS, Subramanyam MA, Nair NS, Rajashekhar B. Hearing impairment and ear diseases among children of school entry age in rural south India. Int J Pediatr Otorhinolaryngol 2002;64:105-10

22 Gell FM, White E, Newell K, Mackenzie I, Smith A, Thompson S. Practical screening priorities for hearing impairment among children in developing countries. Bull World Health Organ 1992;70:645-55

23 Elahi MM, Elahi F, Elahi A, Elahi SB. Paediatric hearing loss in rural Pakistan. J Otolaryngol 1998;27 $348-53$

24 Fortnum H, Davis A. Epidemiology of permanent childhood hearing impairment in the Trent region, 1985-1993. Br J Audiol 1997;31:409-46
Address for correspondence:

Dr I Mackenzie,

Child and Reproductive Health Group,

Liverpool School of Tropical Medicine,

Pembroke Place,

Liverpool L3 5QA, UK.

Fax: +44 (0)151 7053329

E-mail: macken34@liv.ac.uk

Dr I Mackenzie takes responsibility for the integrity of the content of the paper.

Competing interests: None declared 